\title{
MODIFIED EQUATION FOR ADAPTIVE MONOTONE DIFFERENCE SCHEMES AND ITS CONVERGENT ANALYSIS
}

\author{
ZHEN-HUAN TENG
}

\begin{abstract}
A modified parabolic equation for adaptive monotone difference schemes based on equal-arclength mesh, applied to the linear convection equation, is derived and its convergence analysis shows that solutions of the modified equation approach a discontinuous (piecewise smooth) solution of the linear convection equation at order one rate in the $L_{1}$-norm. It is well known that solutions of the monotone schemes with uniform meshes and their modified equation approach the same discontinuous solution at a half-order rate in the $L_{1}$-norm. Therefore, the convergence analysis for the modified equation provided in this work demonstrates theoretically that the monotone schemes with adaptive grids can improve the solution accuracy. Numerical experiments also confirm the theoretical conclusions.
\end{abstract}

\section{INTRODUCTION}

In this paper we consider adaptive monotone difference schemes applied to scalar conservation laws

$$
\left\{\begin{array}{lll}
\frac{\partial u}{\partial t}+\frac{\partial f(u)}{\partial x}=0 & \text { for } & (x, t) \in \mathbf{R} \times \mathbf{R}^{+}, \\
u(x, 0)=u_{0}(x) & \text { for } & x \in \mathbf{R} .
\end{array}\right.
$$

The monotone difference schemes approaching the conservation laws are in the following form:

$$
u_{j}^{n+1}=u_{j}^{n}-\lambda\left(\bar{f}\left(u_{j}^{n}, u_{j+1}^{n}\right)-\bar{f}\left(u_{j-1}^{n}, u_{j}^{n}\right)\right) \equiv H\left(u_{j-1}^{n}, u_{j}^{n}, u_{j+1}^{n}\right),
$$

where $\lambda=\Delta t / \Delta x$ is the Courant number, $\bar{f}(u, u)=f(u)$ is the consistent condition and $H_{i}\left(u_{-1}, u_{0}, u_{1}\right) \equiv \partial_{u_{i}} H\left(u_{-1}, u_{0}, u_{1}\right) \geq 0$ for $i=-1,0,1$ are the monotone conditions. Here $u_{j}^{n}$ are the numerical solutions, which approximate the exact solution $u(x, t)$ at the point $\left(x_{j}, t_{n}\right)$, where $x_{j}$ for $j=0, \pm 1, \pm 2, \ldots$ and $t_{n}$ for $n=0,1,2, \ldots$ are space and time grid points respectively. It is known that the monotone difference schemes are of first-order accuracy and include several popular difference schemes such as the Lax-Friedrichs scheme, the Godunov scheme and the Engquist-Osher scheme.

Received by the editor August 2, 2006 and, in revised form, April 17, 2007.

2000 Mathematics Subject Classification. Primary 65M06, 65M15, 35K15.

Key words and phrases. Error estimates, adaptive monotone schemes, modified parabolic equation, convection equation.

This work was supported in part by the National Natural Science Foundation of China (10576001).

(C)2008 American Mathematical Society Reverts to public domain 28 years from publication 
For uniform mesh, $x_{j}$ is defined by $x_{j}=j \Delta x$ with a uniform space step size $\Delta x$. For adaptive mesh, $x_{j}$ is defined based on the equidistribution principle [5, 11, which can be expressed in integral form as

$$
\int_{x_{j}}^{x_{j+1}} M(x, t) d x=\Delta s,
$$

where $M(x, t)$ is a chosen monitor function and $\Delta s$ is a uniform step size in the computational domain. In this paper we will consider the popular arclength monitor function,

$$
M(x, t)=\sqrt{1+\partial_{x} u(x, t)^{2}},
$$

where $u$ is the solution of the underlying PDE. Therefore, $x_{j}$ is defined based on the equal-arclength principle by

$$
\int_{x_{j}}^{x_{j+1}} \sqrt{1+\left(\partial_{x} u(x, t)\right)^{2}} d x=\Delta s,
$$

where $\Delta s$ is an arc-length step size. Let $\Delta x_{i}=x_{i+1}-x_{i}$, then

$$
\Delta x_{i}=\frac{\Delta s}{\sqrt{1+\left(\partial_{x} u\left(\tilde{x}_{i}, t\right)\right)^{2}}},
$$

where $\tilde{x}_{i}$ is located between $x_{i}$ and $x_{i+1}$.

A truncation error analysis shows that the modified equation for uniform monotone difference schemes (1.2) is given in the form 4 ]

$$
\frac{\partial v_{\Delta x}}{\partial t}+\frac{\partial f\left(v_{\Delta x}\right)}{\partial x}=\Delta x \frac{\partial}{\partial x}\left[\beta\left(v_{\Delta x}, \lambda\right) \frac{\partial v_{\Delta x}}{\partial x}\right],
$$

where

$$
\beta(v, \lambda)=\lambda\left[\frac{1}{2 \lambda^{2}} \sum_{k=-1}^{1} j^{2} H_{j}(v, v, v)-\frac{1}{2} a^{2}(v)\right] \geq 0,
$$

$a(v)=f^{\prime}(v)$ and $H_{j}\left(v_{-1}, v_{0}, v_{1}\right)=\partial_{v_{j}} H\left(v_{-1}, v_{0}, v_{1}\right)$ for $j=-1,0,1$. It is widely believed that solutions of uniform monotone schemes such as (1.2) often behave very much like solutions of the modified parabolic equation (1.6).

If the modified equation is applied to an adaptive interval $\left[x_{j}, x_{j+1}\right]$, then it results in

$$
\frac{\partial w_{\Delta s}}{\partial t}+\frac{\partial f\left(w_{\Delta s}\right)}{\partial x}=\Delta x_{i} \frac{\partial}{\partial x}\left[\beta\left(w_{\Delta s}, \lambda\right) \frac{\partial w_{\Delta s}}{\partial x}\right] .
$$

Substituting $\Delta x_{i}=\Delta s / \sqrt{1+\left(\partial_{x} w_{\Delta s}\left(\tilde{x}_{i}, t\right)\right)^{2}}$ into the above equation and setting the $\tilde{x}_{i}=x$ gives

$$
\frac{\partial w_{\Delta s}}{\partial t}+\frac{\partial f\left(w_{\Delta s}\right)}{\partial x}=\frac{\Delta s}{\sqrt{1+\left(\partial_{x} w_{\Delta s}\right)^{2}}} \frac{\partial}{\partial x}\left[\beta\left(w_{\Delta s}, \lambda\right) \frac{\partial w_{\Delta s}}{\partial x}\right] .
$$

This is a modified parabolic equation for adaptive monotone difference schemes based on equal-arclength mesh. Thus, we may believe that the adaptive modified parabolic equation and the adaptive monotone schemes should share many important properties and their solutions should be in agreement as well.

It is well known that for the linear convection equation, the uniform monotone schemes approaching piecewise smooth solution with a finite number of discontinuous curves have only half-order rate of convergence in the $L_{1}$-norm 6, 1]. More precisely, let $u_{\Delta x}(x, t)$ be the numerical solutions of uniform monotone schemes 
(1.2) with initial data $u_{\Delta x}(x, 0)=u_{0}(x)$ and let $u(x, t)$ be the piecewise smooth entropy solution of (1.1) with a finite number of discontinuous curves. Then we have

$$
\left\|u_{\Delta x}(\cdot, t)-u(\cdot, t)\right\|_{L_{1}} \leq C(t \Delta x)^{1 / 2} \text { for } f(u)=a u,
$$

where $a$ is a constant. Similarly, for the modified equation of uniform monotone schemes we have the same error estimate

$$
\left\|v_{\Delta x}(\cdot, t)-u(\cdot, t)\right\|_{L_{1}} \leq C(t \Delta x)^{1 / 2} \text { for } f(u)=a u,
$$

where $v_{\Delta x}(\cdot, t)$ is the solution of (1.6) with initial data $v_{\Delta x}(x, 0)=u_{0}(x)$.

We have to point out that the half-order rates of convergence for the linear convection equation given in (1.9) and (1.10) are the best possible [12, 13]. But for nonlinear conservation law, i.e., $f^{\prime \prime}(u) \neq 0$, the half-order rates of convergence can be improved to one [13, 14, 2].

The interesting question arises as to whether the adaptive monotone schemes and their modified equation for linear convection equation improve the rate of convergence. In this paper we will answer the second part of the questions and show that for the adaptive modified equation the convergent rate indeed increases from $1 / 2$ to 1 for the linear case. More precisely, we have proved that

$$
\left\|w_{\Delta s}(\cdot, t)-u(\cdot, t)\right\|_{L_{1}} \leq C(t \Delta s)|\ln (t \Delta s)| \text { for } f(u)=a u,
$$

where $w_{\Delta s}(\cdot, t)$ is the solution of the adaptive modified equation (1.8) with initial data $w_{\Delta x}(x, 0)=u_{0}(x)$. The above result is a theoretical indicator that the adaptive monotone schemes for the linear convection equation improve the rate of convergence. We also carry out numerical experiments using adaptive upwind monotone scheme to compute a discontinuous solution of the linear convection equation and the result confirms that its convergent rate in the $L^{1}$-norm indeed approaches one.

\section{LINEAR CONVECTION EQUATION}

For linear convection equation

$$
\begin{cases}\frac{\partial u}{\partial t}+a \frac{\partial u}{\partial x}=0, & (x, t) \in \mathbf{R} \times \mathbf{R}^{+} \\ u(x, 0)=u_{0}(x), & x \in \mathbf{R},\end{cases}
$$

where $a$ is a constant, linear monotone schemes take the form

$$
u_{j}^{n+1}=\lambda(\gamma-a / 2) u_{j+1}^{n}+(1-2 \gamma \lambda) u_{j}^{n}+\lambda(\gamma+a / 2) u_{j-1}^{n},
$$

where $\gamma$ is a constant, $\lambda$ is the Courant number and they satisfy monotone conditions:

$$
\gamma \geq|a| / 2 \text { and } \lambda \leq \frac{1}{2 \gamma} .
$$

The modified equation (1.6) for uniform monotone schemes, applied to the linear equation (2.1), is in the form

$$
\begin{cases}\frac{\partial v_{\Delta s}}{\partial t}+a \frac{\partial v_{\Delta s}}{\partial x}=\Delta x \frac{\partial}{\partial x}\left[\beta(\lambda) \frac{\partial v_{\Delta s}}{\partial x}\right], & (x, t) \in \mathbf{R} \times \mathbf{R}^{+}, \\ v_{\Delta s}(x, 0)=u_{0}(x), & x \in \mathbf{R},\end{cases}
$$

where

$$
\beta(\lambda)=\gamma-\frac{\lambda}{2} a^{2} \geq \frac{|a|}{2}(1-\lambda|a|) \geq 0,
$$


which is independent of $v_{\Delta x}$. Hence the modified equation of uniform monotone schemes is a convection-diffusion equation with a linear viscous term.

On the other hand, the modified equation (1.8) for adaptive monotone schemes based on equal-arclength, applied to the linear equation (2.1), is in the form

$$
\begin{cases}\frac{\partial w_{\Delta s}}{\partial t}+a \frac{\partial w_{\Delta s}}{\partial x}=\frac{\Delta s}{\sqrt{1+\left(\partial_{x} w_{\Delta s}\right)^{2}}} \frac{\partial}{\partial x}\left[\beta(\lambda) \frac{\partial w_{\Delta s}}{\partial x}\right], & (x, t) \in \mathbf{R} \times \mathbf{R}^{+}, \\ w_{\Delta s}(x, 0)=u_{0}(x), & x \in \mathbf{R} .\end{cases}
$$

The above equation can be written as

$$
\begin{cases}\frac{\partial w_{\Delta s}}{\partial t}+a \frac{\partial w_{\Delta s}}{\partial x}=\Delta s \frac{\partial \sigma\left(\partial_{x} w_{\Delta s}\right)}{\partial x}, & (x, t) \in \mathbf{R} \times \mathbf{R}^{+} \\ w_{\Delta s}(x, 0)=u_{0}(x), & x \in \mathbf{R} .\end{cases}
$$

where $\sigma(w)$ is defined by

$$
\sigma(w)=\beta(\lambda) \int_{0}^{w} \frac{1}{\sqrt{1+w^{2}}} d w=\beta(\lambda) \ln \left(w+\sqrt{1+w^{2}}\right) .
$$

Notice that the adaptive modified equation (2.5) is a convection-diffusion equation with a nonlinear viscous term, which is a quasilinear parabolic equation in divergence form. Several books (see for example [7, 8]) are devoted to the existence of solutions of such problems. In this paper we will assume that there exists a solution $w_{\Delta s}(x, t)$ of (2.5), which is smooth for $t>0$.

In this paper we will prove the following error estimate theorem.

Theorem 2.1. If $u_{0}(x)$ is a piecewise smooth function with a finite number of discontinuous points and $u$ and $w_{\Delta s}$ are solutions of (2.1) and (2.5) respectively, then we have

$$
\left\|w_{\Delta s}(\cdot, t)-u(\cdot, t)\right\|_{L_{1}} \leq C t \Delta s(1+|\ln (t \Delta s)|),
$$

where $C$ is a constant depending only on the initial data $u_{0}$, but it is independent of $t$ and $\Delta s$.

In what follows $C$ stands for a constant only depending on $u_{0}$, but $C$ may stand for different constants at different places.

Since variable substitution $\xi=x-$ at can change (2.5) into

$$
\begin{cases}\frac{\partial \bar{w}_{\Delta s}}{\partial t}=\Delta s \frac{\partial \sigma\left(\partial_{\xi} \bar{w}_{\Delta s}\right)}{\partial \xi}, & (\xi, t) \in \mathbf{R} \times \mathbf{R}^{+}, \\ \bar{w}_{\Delta s}(\xi, 0)=u_{0}(\xi), & \xi \in \mathbf{R},\end{cases}
$$

the solution $w_{\Delta s}(x, t)$ of (2.5) can be expressed as

$$
w_{\Delta s}(x, t)=\bar{w}_{\Delta s}(x-a t, t),
$$

where $\bar{w}_{\Delta s}(\xi, t)$ is the solution of (2.8). Therefore, in this paper we mainly consider the case $a=0$ and will prove Theorem 2.1 for the special case. For the general case of $a \neq 0$, the expression (2.9) will help in obtaining the same error estimate (2.7).

\section{Proof of Theorem 2.1}

In this section we will prove the main result of Theorem 2.1. The first-order rate of convergence for the adaptive modified equation is established by using properties of an auxiliary similarity solution and a stability result of the adaptive modified equation. 
3.1. Auxiliary viscous equation and auxiliary function. Without loss of generality, we will assume that $u_{0}(x)$ has only one discontinuous point at $x=0$. Therefore, the solution $u(x, t)$ of (2.1) has only one discontinuous line along $x=a t$. In fact $u(x, t)$ of linear convection equation (2.1) can be expressed as

$$
u(x, t)=u_{0}(x-a t) .
$$

Notice that in the case of $a=0$,

$$
u(x, t)=u_{0}(x) .
$$

We consider the following Riemann initial value problem for an auxiliary viscous equation

$$
\begin{cases}\frac{\partial W_{\Delta s}}{\partial t}=\Delta s \beta(\lambda)\left|\frac{\partial W_{\Delta s}}{\partial x}\right|^{-1} \frac{\partial^{2} W_{\Delta s}}{\partial x^{2}}, & (x, t) \in \mathbf{R} \times \mathbf{R}^{+}, \\ W_{\Delta s}(x, 0)=U^{(0)}\left(x ; u_{-}, u_{+}\right), & x \in \mathbf{R},\end{cases}
$$

where $U^{(0)}\left(x ; u_{-}, u_{+}\right)$is defined by

$$
U^{(0)}\left(x ; u_{-}, u_{+}\right)= \begin{cases}u_{-}, & x \leq 0 \\ u_{+}, & x>0\end{cases}
$$

with $u_{ \pm}=\lim _{x \rightarrow \pm 0} u_{0}(x)$.

The Riemann problem (3.2) has an explicit similarity solution. Letting $W_{\Delta s}(x, t)$ $=Z(x /(t \Delta s))$ and substituting it into (3.2) gives

$$
\left\{\begin{array}{l}
-\left(Z^{\prime}(\eta)\right)^{2} \eta=\beta(\lambda) Z^{\prime \prime}(\eta) \\
Z( \pm \infty)=u_{ \pm}
\end{array}\right.
$$

where $\eta=x /(t \Delta s)$. Solving (3.4) for $Z$ gives

$$
Z(\eta)=\frac{u_{+}-u_{-}}{\pi} \arctan \left(\frac{\left(u_{+}-u_{-}\right) \eta}{2 \beta(\lambda) \pi}\right)+\frac{u_{+}+u_{-}}{2} .
$$

Thus the similarity solution is

$$
W_{\Delta s}(x, t)=Z(x /(t \Delta s))=\frac{u_{+}-u_{-}}{\pi} \arctan \left(\frac{u_{+}-u_{-}}{2 \beta(\lambda) \pi} \frac{x}{t \Delta s}\right)+\frac{u_{+}+u_{-}}{2} .
$$

The following lemma is an easy conclusion from the above expression.

Lemma 3.1. For the Riemann solution $W_{\Delta s}(x, t)$ of (3.2) and its initial data $U^{(0)}\left(x ; u_{-}, u_{+}\right)$of (3.3), we have

$$
\left\|W_{\Delta s}(\cdot, t)-U^{(0)}\left(\cdot ; u_{-}, u_{+}\right)\right\|_{L_{1}}=C t \Delta s,
$$

where $C=\left\|Z(\cdot)-U^{(0)}\left(\cdot ; u_{-}, u_{+}\right)\right\|_{L_{1}}$ and $Z(\eta)$ is defined by (3.5).

Proof. Using the similarity of the Riemann solution (3.6), we have

$$
\begin{aligned}
& \left\|W_{\Delta s}(\cdot, t)-U^{(0)}\left(\cdot ; u_{-}, u_{+}\right)\right\|_{L_{1}}=\left\|Z(\cdot /(t \Delta s))-U^{(0)}\left(\cdot ; u_{-}, u_{+}\right)\right\|_{L_{1}} \\
& =t \Delta s\left\|Z(\cdot)-U^{(0)}\left(\cdot ; u_{-}, u_{+}\right)\right\|_{L_{1}}=C t \Delta s,
\end{aligned}
$$

where we apply the $W_{\Delta s}(x, t)$ expression (3.6) to the first integration to obtain the first equation and use the rescale substitution in the second integration to obtain the third one. 
We construct an auxiliary function $V_{\Delta s}(x, t)$ :

$$
V_{\Delta s}(x, t)=u(x, t)-U^{(0)}\left(x ; u_{-}, u_{+}\right)+W_{\Delta s}(x, t) .
$$

Substituting (3.1) into the above equation gives

$$
V_{\Delta s}(x, t)=u_{0}(x)-U^{(0)}\left(x ; u_{-}, u_{+}\right)+W_{\Delta s}(x, t) .
$$

Remark 3.2. The auxiliary function $V_{\Delta s}(x, t)$ is a continuous function on $\mathbf{R} \times(0, \infty)$, but $\partial_{x} V_{\Delta s}(x, t)$ has a discontinues line along $x=0$.

We can write $u(x, t)-w_{\Delta s}(x, t)=u(x, t)-V_{\Delta s}(x, t)+V_{\Delta s}(x, t)-w_{\Delta s}(x, t)$ and use the triangular inequality:

$$
\left\|u(\cdot, t)-w_{\Delta s}(\cdot, t)\right\|_{L_{1}} \leq\left\|u(\cdot, t)-V_{\Delta s}(\cdot, t)\right\|_{L_{1}}+\left\|V_{\Delta s}(\cdot, t)-w_{\Delta s}(\cdot, t)\right\|_{L_{1}} .
$$

It follows from (3.8) and (3.7) that

$$
\left\|u(\cdot, t)-V_{\Delta s}(\cdot, t)\right\|_{L_{1}}=\left\|U^{(0)}\left(\cdot ; u_{-}, u_{+}\right)-W_{\Delta s}(\cdot, t)\right\|_{L_{1}}=C t \Delta s .
$$

Using the stable property of the viscous equation (2.5), We will show:

$$
\left\|V_{\Delta s}(\cdot, t)-w_{\Delta s}(\cdot, t)\right\|_{L_{1}} \leq C t \Delta s(1+|\ln (t \Delta s)|) .
$$

Thus the main result (2.7) follows from (3.10), (3.11) and (3.12). The estimate (3.12) will be carried out in the next two subsections.

3.2. Stability theorem for the adaptive modified equation. The following stability of the adaptive modified equation (2.5) plays an important role in the estimate of (3.12).

Theorem 3.3. Let $w$ be a viscous solution of (2.5). Let $v \in C(\boldsymbol{R} \times(0, \infty))$ be a piecewise smooth function having derivative jumps along some curves $x=$ $Y_{i}(t), 0 \leq i \leq I$. If $v$ satisfies

$$
\frac{\partial}{\partial t} v+a \frac{\partial}{\partial x} v=\Delta s \frac{\partial}{\partial x} \sigma\left(\partial_{x} v\right)+g(x, t)
$$

everywhere except on the curves $x=Y_{i}(t), 0 \leq i \leq I$, then for any $0 \leq \tau<t<\infty$,

$$
\begin{aligned}
& \|w(\cdot, t)-v(\cdot, t)\|_{L^{1}(\mathbf{R})} \leq\|w(\cdot, \tau)-v(\cdot, \tau)\|_{L^{1}(\mathbf{R})} \\
& \quad+\Delta s \sum_{i=0}^{I} \int_{\tau}^{t}\left|\left[\sigma\left(\partial_{x} v(x, t)\right)\right]_{Y_{i}(t)}\right| d t+\int_{\tau}^{t}\|g(\cdot, t)\|_{L^{1}} d t,
\end{aligned}
$$

where the jumps are defined by

$$
[\rho(x, t)]_{Y(t)}:=\rho(Y(t)+0, t)-\rho(Y(t)-0, t)
$$

Proof. Without loss of generality we assume that there is only one curve $x=Y_{0}(t)$. We write

$$
\|w(\cdot, t)-v(\cdot, t)\|_{L^{1}(\mathbf{R})}=\sum_{j=-L}^{K}(-1)^{j} \int_{y_{j}}^{y_{j+1}}(w(x, t)-v(x, t)) d x,
$$

where $w-v$ changes sign at the points $y_{j}=y_{j}(t)$ for $j=-L, \cdots, K$, with the assumption of $y_{-L}=-\infty$ and $y_{K+1}=+\infty$.

Since $w$ and $v$ are continuous,

$$
\left.(w(x, t)-v(x, t))\right|_{x=y_{j}}=0 \quad \text { for all } j .
$$


Differentiating (3.15) and using (3.16) gives

$$
\frac{d}{d t}\|w(\cdot, t)-v(\cdot, t)\|_{L^{1}(\mathbf{R})}=\sum_{j=-L}^{K}(-1)^{j} \int_{y_{j}}^{y_{j+1}} \frac{\partial}{\partial t}(w(x, t)-v(x, t)) d x .
$$

Substituting (2.5) and (3.13) into the above equation and carrying out the integrations, we obtain

$$
\begin{aligned}
\frac{d}{d t}\|w(\cdot, t)-v(\cdot, t)\|_{L^{1}(\mathbf{R})} & \leq-\left.a \sum_{j=-L}^{K}(-1)^{j}(w(x, t)-v(x, t))\right|_{y_{j}} ^{y_{j+1}} \\
& +\left.\sum_{j=-L}^{K}(-1)^{j} \Delta s\left(\sigma\left(w_{x}(x, t)\right)-\sigma\left(v_{x}(x, t)\right)\right)\right|_{y_{j}} ^{y_{j+1}} \\
& +\Delta s\left|\left[\sigma\left(v_{x}(x, t)\right)\right]_{Y_{0}}\right|+\|g(\cdot, t)\|_{L^{1}} .
\end{aligned}
$$

On account of (3.16), the first term on the right of the above inequality is zero. Since $v_{x}$ has a jump at $Y_{0}$ the third term on the right appears. We now prove that each summand in the sum given in the second term is nonpositive. Since $(-1)^{j}(w-v)=p \geq 0$ for $x \in\left[y_{j}, y_{j+1}\right]$ and zero at the endpoints, $\left.p_{x}\right|_{y_{j}} \geq 0$ and $\left.p_{x}\right|_{y_{j+1}} \leq 0$. Therefore, the summand in the sum can be expressed as

$$
\left.(-1)^{j} \Delta s \sigma^{\prime}(\tilde{z})(w(x, t)-v(x, t))_{x}\right|_{y_{j}} ^{y_{j+1}}=\left.\Delta s \sigma^{\prime}(\tilde{z}) p_{x}\right|_{y_{j}} ^{y_{j+1}} \leq 0
$$

where we have used the fact that $\sigma^{\prime}(\tilde{z})=1 /\left(1+\tilde{z}^{2}\right)>0$. Thus integrating the inequality (3.18) with respect to $t$ from $\tau$ to $t$ yields the stable inequality (3.14).

3.3. Estimate of $\left\|V_{\Delta s}(\cdot, t)-w_{\Delta s}(\cdot, t)\right\|_{L_{1}}$. It follows from the definition (3.9) that

$$
V_{\Delta s}(x, t)=u_{0}(x)-U^{(0)}\left(x ; u_{-}, u_{+}\right)+W_{\Delta s}(x, t) \in C(\mathbf{R} \times(0, \infty)),
$$

its derivatives have jumps along the discontinues curve $x=0$ and $V_{\Delta s}$ satisfies

$$
\frac{\partial}{\partial t} V_{\Delta s}=\Delta s \frac{\partial}{\partial x} \sigma\left(\partial_{x} V_{\Delta s}\right)+g(x, t) \quad \text { for } x \neq 0
$$

with

$$
g(x, t)=\frac{\partial}{\partial t} W_{\Delta s}-\Delta s \frac{\partial}{\partial x} \sigma\left(\partial_{x}\left(u_{0}+W_{\Delta s}\right)\right) .
$$

Therefore, we can apply Theorem 3.3 to $V_{\Delta s}$ and $w_{\Delta s}$ and use the inequality (3.14) for $I=0, Y_{0}(t)=0$ and $\tau=0$, i.e.,

$$
\begin{array}{r}
\left\|w_{\Delta s}(\cdot, t)-V_{\Delta s}(\cdot, t)\right\|_{L^{1}(\mathbf{R})} \leq\left\|w_{\Delta s}(\cdot, 0)-V_{\Delta s}(\cdot, 0)\right\|_{L^{1}(\mathbf{R})} \\
\quad+\Delta s \int_{0}^{t}\left|\left[\sigma\left(\partial_{x} V_{\Delta s}(x, t)\right)\right]_{x=0}\right| d t+\int_{0}^{t}\|g(\cdot, t)\|_{L^{1}} d t,
\end{array}
$$

to prove the estimate (3.12).

It follows from the definition (3.9) and from the initial data (2.5) and (3.2) that the first term on the right-hand side of (3.21) is zero. We begin to estimate the second term on the right:

$$
\Delta s \int_{0}^{t}\left|\left[\sigma\left(\partial_{x} V_{\Delta x}(x, t)\right)\right]_{x=0}\right| d t=\Delta s \int_{0}^{t}\left|\left[\sigma\left(u_{0}^{\prime}(x)\right)\right]_{x=0}\right| d t \leq C t \Delta s,
$$

where $C=2 \beta(\lambda) \max \left\{\left|u_{0}^{\prime}(+0)\right|,\left|u_{0}^{\prime}(-0)\right|\right\}$. 
Substituting the equation (3.2) into the first term on the right-hand side of (3.20) and carrying out some calculation gives

$$
\begin{aligned}
g(x, t) & =\frac{\Delta s u_{0}^{\prime \prime}}{\sqrt{1+\left(u_{0}^{\prime}+\partial_{x} W_{\Delta s}\right)^{2}}} \\
& +\frac{\Delta s\left(1+u_{0}^{\prime 2}\right) \partial_{x}^{2} W_{\Delta s}}{\sqrt{1+\left(u_{0}^{\prime}+\partial_{x} W_{\Delta s}\right)^{2}}\left|\partial_{x} W_{\Delta s}\right|\left(1+\left(\partial_{x} W_{\Delta s}\right)^{2}+\left(u_{0}^{\prime}+\partial_{x} W_{\Delta s}\right)^{2}\right)} \\
& +\frac{2 \Delta s u_{0}^{\prime} \partial_{x}^{2} W_{\Delta s}}{\sqrt{1+\left(u_{0}^{\prime}+\partial_{x} W_{\Delta s}\right)^{2}}\left(1+\left(\partial_{x} W_{\Delta s}\right)^{2}+\left(u_{0}^{\prime}+\partial_{x} W_{\Delta s}\right)^{2}\right)} \\
& :=I+I I+I I I .
\end{aligned}
$$

It is easy to show that

$$
\|I\|_{L^{1}} \leq \Delta s\left\|u_{0}^{\prime \prime}(\cdot)\right\|_{L^{1}}
$$

where the integration means $\left\|u_{0}^{\prime \prime}(\cdot)\right\|_{L^{1}}=\int_{-\infty}^{0}+\int_{0}^{\infty}\left|u^{\prime \prime}(x)\right| d x$. It follows from the definition of $I I$ that

$$
\|I I\|_{L^{1}} \leq C \Delta s \int_{-\infty}^{\infty}\left|\frac{\partial_{x}^{2} W_{\Delta s}(x, t)}{\partial_{x} W_{\Delta s}(x, t)}\right| d x \leq C \Delta s 2 \sup _{x}|\ln | \partial_{x} W_{\Delta s}(x, t) \|,
$$

where $C=1+\sup _{x} u_{0}^{\prime}(x)^{2}$. Since $\partial_{x} W_{\Delta s}(x, t)$ does not change its sign and $\partial_{x}^{2} W_{\Delta s}(x, t)$ only changes its sign once for $x \in(-\infty, \infty)$, the above integration can be integrated out and gives the last inequality. Differentiating (3.6) with respect to $x$, we obtain

$$
\begin{aligned}
\left|\partial_{x} W_{\Delta s}(x, t)\right| & =\left|\frac{u_{+}-u_{-}}{\pi}\left(1+\left(\frac{u_{+}-u_{-}}{2 \beta(\lambda) \pi} \frac{x}{t \Delta s}\right)^{2}\right)^{-1} \frac{u_{+}-u_{-}}{2 \beta(\lambda) \pi} \frac{1}{t \Delta s}\right| \\
& \leq \frac{\left(u_{+}-u_{-}\right)^{2}}{2 \beta(\lambda) \pi^{2}} \frac{1}{t \Delta s}=C \frac{1}{t \Delta s} .
\end{aligned}
$$

Substituting the inequality into (3.24) gives

$$
\|I I\|_{L^{1}} \leq C \Delta s(1+|\ln (t \Delta s)|)
$$

Similarly, it follows from the definition of III that

$$
\begin{aligned}
\|I I I\|_{L^{1}} & \leq 2 \Delta s \sup _{x}\left|u_{0}^{\prime}\right| \int_{-\infty}^{\infty} \frac{\left|\partial_{x}^{2} W_{\Delta s}\right|}{1+\left(\partial_{x} W_{\Delta s}\right)^{2}} d x \\
& \leq 2 \Delta s \sup _{x}\left|u_{0}^{\prime}\right| 2 \sup _{x}\left|\arctan \left(\partial_{x} W_{\Delta s}\right)\right|=2 \pi \sup _{x}\left|u_{0}^{\prime}\right| \Delta s .
\end{aligned}
$$

Adding up (3.23), (3.26) and (3.27) and integrating from 0 to $t$ gives

$$
\begin{aligned}
\int_{0}^{t}\|g(\cdot, t)\|_{L^{1}} d t & \leq \int_{0}^{t}\left(\|I\|_{L^{1}}+\|I I\|_{L^{1}}+\|I I I\|_{L^{1}}\right) d t \\
& \leq C \Delta s \int_{0}^{t}(1+\ln (t \Delta s)) d t \leq C t \Delta s(1+|\ln (t \Delta s)|) .
\end{aligned}
$$

Thus the important estimate (3.12) follows from (3.21), (3.22) and (3.28). Now we have completed the proof of Theorem 2.1 . 


\section{NUMERICAL EXPERIMENTS}

In this section we will compute a discontinuous solution of the following linear convection equation using the adaptive upwind monotone difference scheme and show numerically that its $L^{1}$-convergent rate is one. The linear convection equation is

$$
\begin{cases}\frac{\partial u}{\partial t}+\frac{\partial u}{\partial x}=0 & \text { for } \quad(x, t) \in \mathbf{R} \times \mathbf{R}^{+} \\ u(x, 0)=u_{0}(x) & \text { for } \quad x \in \mathbf{R}\end{cases}
$$

where $u_{0}$ is a periodic function with period $\pi$, which is defined by

$$
u_{0}(x)=\operatorname{sign}(x-\pi / 4) \sin (x) \text { for } x \in(0, \pi]
$$

Notice that $u_{0}$ has a discontinuous point at $x=\pi / 4$. The exact solution of (4.1) is $u(x, t)=u_{0}(x-t)$, which has a discontinuous curve $x=\pi / 4+t$. The discontinuous solution is computed by the following adaptive upwind monotone scheme for $x \in \mathbf{R}$ :

$$
\left\{\begin{array}{l}
\frac{u_{\Delta s}^{n+1}(x)-u_{\Delta s}^{n}(x)}{\Delta t}+\frac{u_{\Delta s}^{n}(x)-u_{\Delta s}^{n}(x-\Delta x)}{\Delta x}=0 \\
u_{\Delta s}^{0}=u_{0}(x)
\end{array}\right.
$$

where the adaptive space step $\Delta x$ is defined by

$$
\int_{x-\Delta x}^{x} M\left(D_{x} u_{\Delta s}^{n}(x)\right) d x=\Delta s .
$$

Here $\Delta s$ is a uniform arclength step size, $M(\omega)$ is the equal-arclength monitor function

$$
M(\omega)=\sqrt{1+\omega^{2}}
$$

and $D_{x} u_{\Delta s}^{n}(x)$ is some kind approximation of $\partial_{x} u_{\Delta s}^{n}(x)$ given below. From the definition (4.4) we see that $\Delta x$ is a time $t_{n}$ and space $x$ dependent function.

Notice that the scheme (4.3) is continuously defined at every space point $x \in \mathbf{R}$. The main advantage of such a scheme is that we can, in principle, evolve the numerical solution from $t_{n}$ to $t_{n+1}$ directly without the need of any mesh redistribution and solution interpolation being carried out at each time level, which usually is a necessary step for traditional adaptive schemes. Thus the scheme can avoid any numerical pollution from this kind of interpolation and is more closely related to the modified equation (2.5). In the following we will show numerically that the adaptive upwind monotone scheme based on the formulation (4.3) indeed achieves a first-order rate of convergence.

In practice, the adaptive upwind scheme (4.3) is solved on a very fine uniform space mesh $x_{l}=l \delta x$ for $l=0, \cdots, L$ :

$$
\left\{\begin{array}{l}
\frac{u_{\Delta s}^{n+1}\left(x_{l}\right)-u_{\Delta s}^{n}\left(x_{l}\right)}{\Delta t}+\frac{u_{\Delta s}^{n}\left(x_{l}\right)-u_{\Delta s}^{n}\left(x_{l}-\Delta x_{l}\right)}{\Delta x_{l}}=0, \\
u_{\Delta s}^{0}\left(x_{l}\right)=u_{0}\left(x_{l}\right),
\end{array}\right.
$$

where the adaptive space step $\Delta x_{l}$ is defined by a discrete formula of the integration (4.4) given below and $D_{x} u_{\Delta s}^{n}\left(x_{l}\right)$ is defined by a backward difference quotient:

$$
D_{x} u_{\Delta s}^{n}\left(x_{l}\right)=\frac{u_{\Delta s}^{n}\left(x_{l}\right)-u_{\Delta s}^{n}\left(x_{l-1}\right)}{\delta x} .
$$


Let the integer $m_{l}$ satisfy

$$
\delta x \sum_{m=0}^{m_{l}} M\left(D_{x} u_{\Delta s}^{n}\left(x_{l-m}\right)\right) \leq \Delta s<\delta x \sum_{m=0}^{m_{l}+1} M\left(D_{x} u_{\Delta s}^{n}\left(x_{l-m}\right)\right)
$$

and then $\Delta x_{l}$ is defined by

$$
\Delta x_{l}=m_{l} \delta x .
$$

Furthermore, the $\Delta s$ and $\delta x$ are defined by

$$
\Delta s=S / J=\left(\int_{0}^{\pi / 4}+\int_{\pi / 4}^{\pi} M\left(u_{0}^{\prime}(x)\right) d x+u_{0}(\pi / 4+0)-u_{0}(\pi / 4-0)\right) / J,
$$

and

$$
\delta x=\pi / L
$$

with $L \gg J$, where $S=5.23441$ is the total arclength of $u_{0}(x)$ for $x$ from 0 to $\pi$ and $L$ and $J$ are some positive integers. In our computation, we set $L=\gamma \cdot J^{2}$, where the integer $\gamma$ is chosen from 0.1 to 1 . It follows from $u(x, t)=u_{0}(x-t)$ that the $S$ is also the total arclength of the solution $u(x, t)$ on the same interval $(0, \pi]$. The time step size $\Delta t$ satisfies the Courant stable condition

$$
\Delta t=\lambda \min _{l} \Delta x_{l}
$$

with $0<\lambda \leq 1$. The adaptive numerical solution $u_{\Delta s}^{n}\left(x_{l}\right)$, with $\lambda=0.8, \gamma=1 / 4$ and $J=20,40,80,160,320,640,1280,2560$, are shown in Table 1 .

TABLE 1. $L^{1}$-error and convergent rate for the adaptive $u_{\Delta s}^{n}\left(x_{l}\right)$ and uniform $v_{j}^{n}$ solution at $t_{n}=\pi / 2$ obtained by the adaptive and uniform upwind scheme respectively.

\begin{tabular}{ccc}
\hline$\Delta s(\Delta x)$ & $L^{1}$-error for $u_{\Delta s}^{n}\left(v_{j}^{n}\right)$ & $L^{1}$-rate for $u_{\Delta s}^{n}\left(v_{j}^{n}\right)$ \\
\hline $2^{-1} S / 10\left(2^{-1} \pi / 6\right)$ & $0.6851357(0.3497294)$ & \\
$2^{-2} S / 10\left(2^{-2} \pi / 6\right)$ & $0.4635859(0.2485957)$ & $0.5635533(0.4924377)$ \\
$2^{-3} S / 10\left(2^{-3} \pi / 6\right)$ & $0.2969826(0.1774331)$ & $0.6424582(0.4865262)$ \\
$2^{-4} S / 10\left(2^{-4} \pi / 6\right)$ & $0.1831727(0.1415126)$ & $0.6971738(0.3263448)$ \\
$2^{-5} S / 10\left(2^{-5} \pi / 6\right)$ & $0.1093112(0.0951744)$ & $0.7447631(0.5722846)$ \\
$2^{-6} S / 10\left(2^{-6} \pi / 6\right)$ & $0.0634865(0.0686452)$ & $0.7839193(0.4714146)$ \\
$2^{-7} S / 10\left(2^{-7} \pi / 6\right)$ & $0.0361822(0.0492286)$ & $0.8111691(0.4796638)$ \\
$2^{-8} S / 10\left(2^{-8} \pi / 6\right)$ & $0.0203097(0.0351111)$ & $0.8331093(0.4875692)$ \\
\hline
\end{tabular}

In order to make a comparison between the adaptive and uniform upwind scheme, we also compute the following uniform upwind scheme:

$$
\left\{\begin{array}{l}
\frac{v_{j}^{n+1}-v_{j}^{n}}{\Delta t}+\frac{v_{j}^{n}-v_{j-1}^{n}}{\Delta x}=0 \\
v_{j}^{0}=u_{0}\left(x_{j}\right)
\end{array}\right.
$$

where $\Delta x$ is uniform space step size, which is independent of $j$ and $n$, and $x_{j}=j \Delta x$. The time step size $\Delta t$ satisfies the Courant stable condition

$$
\Delta t=\lambda \Delta x
$$

with $0<\lambda \leq 1$. The uniform numerical solution $v_{j}^{n}$, with $\lambda=0.8$ and $\Delta x=$ $2^{-1} \pi / 6,2^{-2} \pi / 6, \cdots, 2^{-8} \pi / 6$, are also shown in Table 1. This data, given in Table1, clearly indicates that the rate of convergence for the adaptive upwind scheme 


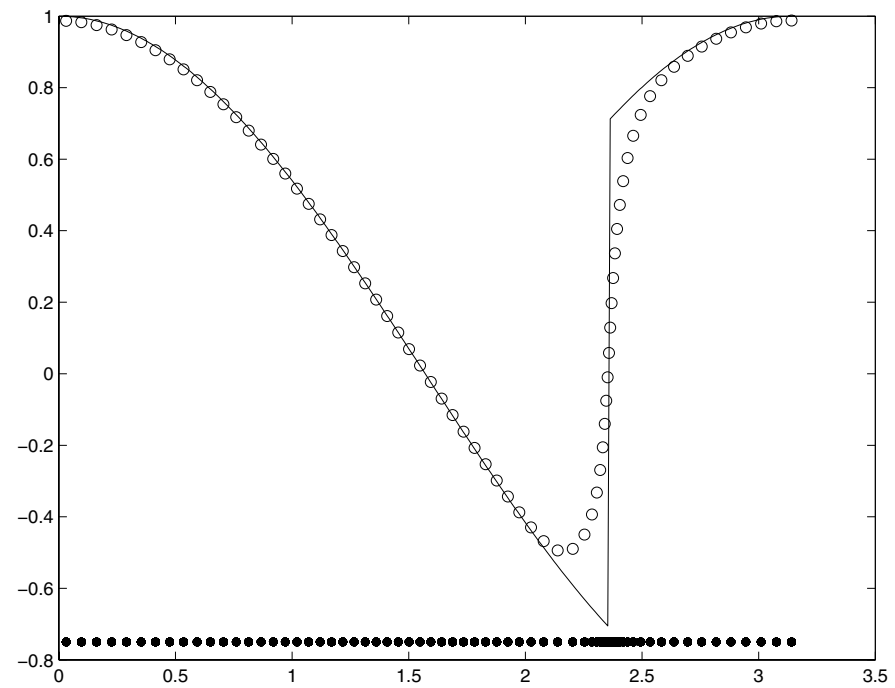

Figure 1. The adaptive numerical "o" and exact "—" solution at $t=\pi / 2$ with $\Delta s=2^{-5} S / 10$, where "•" are the horizontal coordinates of " $\circ$ "

based on (4.5) approaches one and the rate for the uniform upwind scheme (4.6) approaches one half. The adaptive numerical solution and exact solution at $t=\pi / 2$ with $\Delta s=2^{-5} S / 10$ are also plotted in Figure 1, where "o" and "—" represent the numerical and exact solutions respectively. The horizontal coordinates "•" of "०" are given by $x_{l_{j}}$ for $j=0,1,2, \cdots$, which are defined recurrently by $x_{l_{j+1}}=x_{l_{j}}-\Delta x_{l_{j}}$ with $x_{l_{0}}=\pi$. According to the above recurrent formula there are about three hundred points $\left\{x_{l_{j}}\right\}$ located in the interval $(0, \pi]$ and only one fourth of the points are drawn in Figure 1. The figure shows that there are more points clustered in the neighborhood of the discontinuous point $x=3 \pi / 4$.

The adaptive "o" and uniform " $\times$ " upwind solution are plotted in Figure2, From the figure we see that for the adaptive solution more mesh points are crowed round the discontinuous point than that for the uniform solution and also the adaptive solution is closer to the exact solution at the neighborhood of the discontinuous point than the uniform solution.

Before ending this section, we should mention that in recent literature ( 9,10 , 11, 15, 9]) some one-dimensional numerical experiments (similar to the numerical experiment of the present paper) were carried out by adaptive moving mesh methods and the numerical results look better than those given in Figure 1. This is because the one-dimensional problems in $(9,10,11,15,9])$ are initial value problems for nonlinear conservation laws (inviscid Burger's equation), but the problem in this paper is an initial value problem for the linear convection equation (4.1). It is well known that numerical results for nonlinear discontinuity by using finite difference methods are much better than that for linear discontinuity; see for example ([3, 14, 12, 13]). In fact we have already stated in the introduction that the $L_{1}$-convergent rate for nonlinear discontinuity by using the monotone difference schemes is one, but the rate for linear discontinuity is only half. 


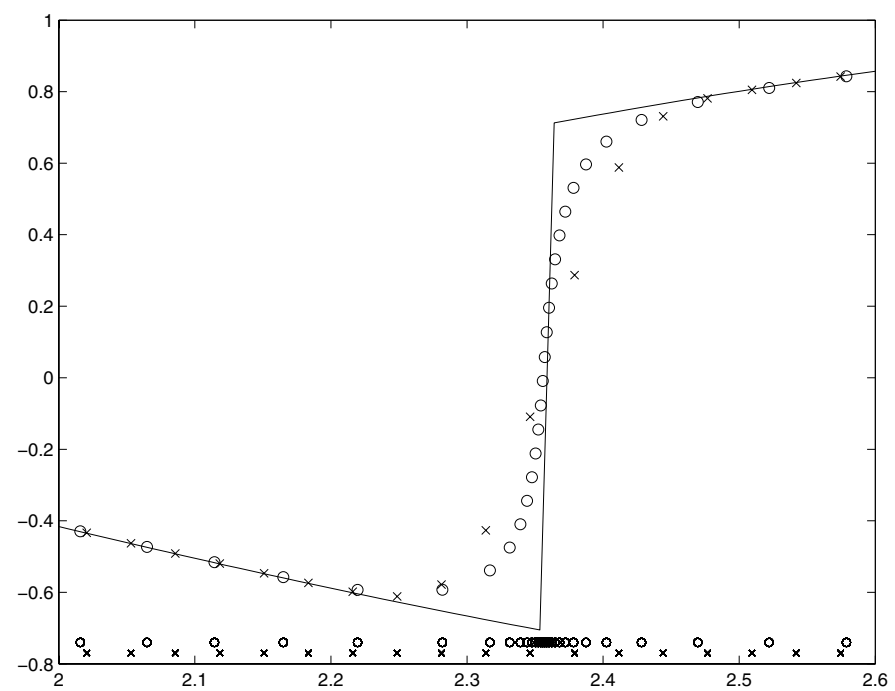

Figure 2. Comparison of the adaptive " $\circ$ " and uniform " $\times$ " solution around the discontinuous point $x=3 \pi / 4$ at $t=\pi / 2$ with $\Delta s=2^{-7} S / 10$ and $\Delta x=2^{-7} \pi / 6$, where the solid line is the exact solution

\section{Concluding Remarks}

To the best of the author's knowledge, there has been very few theoretical results demonstrating the advantages of the time-dependent adaptive grid methods. In this work, we provided a new way to explain high accuracy of these methods by analyzing the resulting modified equation from the adaptive monotone difference schemes. In particular, we have proved the first-order rate of convergence for the resulting modified equation approximating discontinuous solutions. Compared with the adaptive meshes, the optimal rate of convergence for the monotone schemes with fixed meshes and their modified equation is only half. It is noted that the modified equation approach is just a way of explanation; the rigorous first-order rate of convergence for the adaptive monotone difference schemes seems a more challenging problem.

\section{ACKNOWLEDGMENTS}

The author thanks Professor H. Z. Tang of Peking University for many interesting discussions, Professor T. Tang of Hong Kong Baptist University for helpful discussions and useful suggestions, and Dr. H. Y. Jiang of Peking University for helping to draw the figures. He also thanks an anonymous referee for valuable comments and suggestions.

\section{REFERENCES}

[1] F. Bouchut and B. Perthame, Kruzkov's estimates for scalar conservation laws revisited, Trans. Amer. Math. Soc. 350 (1998), 2847-2870. MR1475677 (98m:65156)

[2] B. Despres, Discrete compressive solutions of scalar conservation laws, Journal of Hyperbolic Differential Equations 3 (2004), 493-520. MR2094527(2006a:35194) 
[3] A. Harten, The artificial compression method for computation of shocks and contact discontinuities: III. Self-adjusting hybrid schemes, Math. Comp. 32 (1978), 363-389. MR0489360 $(58: 8789)$

[4] A. Harten, J. M. Hyman and P. D. Lax, On finite-difference approximation and entropy conditions for shocks, Comm. Pure Appl. Math. 29 (1976), 297-322. MR0413526 (54:1640)

[5] W. Huang, Y. Ren and R. D. Russell, Moving mesh partial differential equations (MMPDES) based on the equidistribution principle, SIAM J. Numer. Anal. 31 (1994), 709-730. MR.1275109 (94m:65149)

[6] N. N. Kuznetsov, Accuracy of some approximate methods for computing the weak solutions of a first-order quasi-linear equation, USSR Comp. Math. and Math. Phys. 16 (1976), 105-119.

[7] O. A. Ladyzhenskaja, V. A. Solonnikov and N. N. Ural'ceva, Linear and Quasi-linear Equations of Parabolic Type, Translations of Mathematical Monographs, 23 (1968).

[8] G. M. Leiberman, Second Order Parabolic Differential Equations, World Scientific, (1998).

[9] K. Lipnikov and M. Shashkov, The error-minimization-based strategy for moving mesh methods. Commun. Comput. Phys., 1 (2006), 53-80.

[10] J. M. Stockie, J. A. Mackkenzie and R. D. Russel, A moving mesh method for one-dimensional hyperbolic conservation laws, SIAM J. Sci. Comput. 22 (2001), 1791-1813. MR.1813298 (2001m:65116)

[11] H. Z. Tang and T. Tang, Adaptive mesh methods for one- and two-dimensional hyperbolic conservation laws, SIAM J. Numer. Anal. 41 (2003), 487-515. MR2004185 (2004f:65143)

[12] T. Tang and Z. H. Teng, The sharpness of Kuznetsov's $O(\sqrt{\Delta x}) L^{1}$-error estimate for monotone difference schemes, Math. Comp. 64 (1995), 581-589. MR1270625 (95f:65176)

[13] T. Tang and Z. H. Teng, Viscosity methods for piecewise smooth solutions to scalar conservation laws, Math. Comp. 66 (1997), 495-526. MR1397446 (97m:65160)

[14] Z. H. Teng and P. W. Zhang, Optimal $L^{1}$-rate of convergence for viscosity method and monotone scheme to piecewise constant solutions with shocks, SIAM J. Numer. Anal. 34 (1997), 959-978. MR:1451109 (98f:65094)

[15] Z. Zhang, Moving mesh method with conservative interpolation based on L2-projection, Commun. Comput. Phys. 1 (2006), 930-944.

LMam and School of Mathematical Sciences, Peking University, Beijing 100871, CHINA

E-mail address: tengzh@math.pku.edu.cn 